\title{
Photobiomodulation in Temporomandibular Disorders
}

\author{
Jan Tunér, DDS, ${ }^{1}$ Sepanta Hosseinpour, DDS, MPH, PhD, ${ }^{2}$ and Reza Fekrazad, DDS, PhD, FLD, FICD ${ }^{3,4}$
}

\begin{abstract}
Objective: This systematic review aimed to comprehensively review all available documents regarding photobiomodulation therapy (PBMT) application in temporomandibular disorder (TMD) patients and to suggest an evidence-based protocol for therapeutic PBM administration for these patients.

Background data: The existence of temporomandibular joint and/or pain and dysfunction in masticatory muscles is characterized in TMDs. PBMT is, due to its impact on biological processes, especially inflammation, considered as an adjuvant treatment modality in TMD cases.

Materials and methods: All original articles related to PBMT for TMDs in EMBASE, MEDLINE (NCBI PubMed and PMC), Cochrane library, Scopus, Web of Science, and Google Scholar were reviewed until December 2018.

Results: The energy density ranging from 0.75 to $112.5 \mathrm{~J} / \mathrm{cm}^{2}$ with $0.9-500 \mathrm{~mW}$ power was found to be a window protocol for light application. The best results for pain relief and mandibular movement enhancement were reported after application of GaAlAs diode laser, $800-900 \mathrm{~nm}, 100-500 \mathrm{~mW}$, and $<10 \mathrm{~J} / \mathrm{cm}^{2}$, twice a week for 30 days on trigger points. The session of light applications varied from 1 to 20 .

Conclusions: Although most articles showed that PBMT is effective in reducing pain and contributed to functional enhancement in TMD patients, the heterogenic parameters that have been reported in various studies made the standardization of PBMT complicated. However, such evidence-based consensus can be beneficial for both future research and for clinical applications.
\end{abstract}

Keywords: photobiomodulation, low-level laser therapy, temporomandibular joint, temporomandibular disorder

\section{Introduction}

$\mathbf{C}$ LINICAL DISORDERS OF THE TEMPOROMANDIBULAR JOINT (TMJ) with or without masticatory muscles' disorder encompass as wide range of temporomandibular disorders (TMDs). ${ }^{1}$ Acute or chronic pain, tinnitus, loss of mandibular function, and finally degeneration of tissues are the main signs and symptoms related to TMDs. ${ }^{2-4}$ These issues can be more problematic for patients, influencing quality of life and mental health, ${ }^{5}$ through sleep problems, ${ }^{6}$ increased anxiety and stress, and even causing depression. ${ }^{7}$ The prevalence of TMDs is different between countries (ranges between $21.5 \%$ and $50.5 \%$ ) and genders (more common among males). ${ }^{8-10}$ However, its pathophysiology is still not totally understood. Previous studies have shown that TMD is multi-factorial, encompasses biomechanical, biological and physiological, psychological, and neuromuscular factors. ${ }^{11}$ Therefore, for treating TMDs, various approaches including manual therapy, ${ }^{12}$ ultrasound, ${ }^{13}$ transcutaneous nerve stimulation, ${ }^{14}$ electrotherapy, ${ }^{15}$ and photobiomodulation therapy (PBMT) have been applied. The main purpose of all these therapeutic approaches is to decrease symptom intensity, thereby enhancing the function of the masticatory muscles, TMJ, and adjacent anatomical structures. ${ }^{16-18}$ Among nonsurgical treatment modalities, PBMT has increased in interest during the past few years, conceivably due to its ease of application in conjunction with growing scientific evidence regarding its positive impacts on pain alleviation. ${ }^{19}$

PBMT is a treatment method that commonly consists of noncollimated, coherent, and monochromatic beams of low intensities of laser light. However, during the past decade, noncoherent light sources such as light-emitting diodes (LEDs) have frequently been applied. LEDs do not need the safety considerations like lasers and can be easily administrated

\footnotetext{
${ }^{1}$ Private Practice, Swedish Laser Medical Society (SLMS), Stockholm, Sweden.

${ }^{2}$ School of Dentistry, The University of Queensland, Brisbane, Australia.

${ }^{3}$ Radiation Sciences Research Center, Laser Research Center in Medical Sciences, AJA University of Medical Sciences, Tehran, Iran.

${ }^{4}$ International Network for Photo Medicine and Photo Dynamic Therapy (INPMPDT), Universal Scientific Education and Research Network (USERN), Tehran, Iran.
} 
over a large area of tissues and even at home. ${ }^{20}$ In this review, we searched for all types of PBM administrations but only found one relevant study using LED. ${ }^{21}$ Further, we found one clinical study utilizing a combination of laser and LEDs. ${ }^{22}$

PBM as a nonthermal light can cause metabolic alterations in cells and tissues such as inducing cellular respiration, enhancing vascularization, and fibroblastic differentiation ${ }^{23,24}$ that are important for its therapeutic impacts. Chow and Armati demonstrated that high doses of laser light, in contrast to low stimulatory doses, disrupt neuronal physiology, and the cellular skeleton, and affecting axonal flow that was reversible and did not cause any permanent nerve damage. ${ }^{25}$ The exact mechanism of pain relief through PBMT is not still clear and there is no global consensus regarding "laserassisted analgesia." However, there is some evidence regarding its anti-inflammatory and pan relief impacts in dysfunctional or painful muscles and joints.

One theory suggests the analgesic effect to be a consequence of the reduction of levels of prostaglandin $\mathrm{E},{ }^{26-28}$ which is one of the most important proinflammatory mediators. Moreover, PBM intensifies the synthesis of adenosine triphosphate (ATP), cyclic adenosine monophosphate, and reactive oxygen species. ${ }^{29}$ PBM further increases the electrons' availability in the catalytic center of cyclooxygenase (COX) ${ }^{30-32}$ Several systematic reviews and meta-analyses have analyzed the effect of PBMT in musculoskeletal disorders, but only two partially focused on TMDs. ${ }^{33,34}$ These articles analyzed the effective dosage and power density, related to specific anatomical factors, to assess the reduction of symptoms. ${ }^{33}$

The current systematic review aimed to comprehensively review all available documents regarding PBM application in TMD patients and to suggest a preliminary evidencebased protocol for PBM administration for these patients.

\section{Methods}

\section{Protocol and eligibility criteria}

This review is organized and followed PRISMA guidelines. ${ }^{35}$

Types of studies. All clinical trials, which administrated PBM for TMJ dysfunction as a therapeutic approach and reported the results of PBM individual application, were included. In vivo and in vitro studies, systematic and literature reviews, letter to editors, and theses were omitted.

Types of participants. Humans were the only accepted participants in the included studies, and any type of animal models (such as rats and primates) and high-level laser therapy (such as $\mathrm{CO}_{2}$ laser) were excluded.

Types of interventions. Studies investigating PBMT on TMJ dysfunction including acute/chronic pain, loss of movement, and masticatory malfunction were included.

Types of outcome measures. The included studies were categorized based on the complication types. These complications include pain, mandibular loss of function, and muscular tenderness, which have been evaluated by different tests, such as visual analog scale, ${ }^{36}$ Beck anxiety invento- ry, ${ }^{37}$ colorimetric capsule method, craniomandibular index, and fibromyalgia impact questionnaire.

\section{Information sources}

EMBASE, MEDLINE (NCBI PubMed and PMC), Cochrane library, Scopus, Web of Science, and Google Scholar were the information sources. Moreover, a hand search was performed specifically in the following journals: Lasers in Medical Science, Journal of Photochemistry \& Photobiology B, Photomedicine Laser Surgery, Tissue Engineering: Part A, Lasers in Surgery and Medicine, and Journal of Dental Research.

\section{Search strategy}

An electronic search was conducted in PubMed from January 1990 to December 2018, limited to English language publications. Published articles on photobiomodulation in TMD were found using the following keywords alone or ensemble: ("Laser Therapy" [Mesh] or "Low-Level light Therapy" [Mesh] or "Laser Phototherapy" [Mesh] or "Photobiomodulation Therapy" [Mesh] or "Low-Level Laser Irradiation" [Mesh]) and ("temporomandibular joint disorder" [Mesh] or "craniomandibular disorder" [Mesh] or "temporomandibular joint dysfunction syndrome"[Mesh] or "TMJ pain" or "TMD"). In addition, manual search of the articles in the selected journals was performed.

\section{Study selection and data extraction}

Two independent experienced reviewers analyzed all retrieved articles based on their keywords, titles, and abstracts. After initial assessment, included studies were analyzed based on their full text, and in the case of disagreement between two reviewers, a third expert person involved to resolve it by discussion. After all, we reviewed all full texts in which all the authors participated and confirmed. All steps were performed based on the PRISMA statement. Data were summarized according to the following: (1) author and year of publication; (2) light properties including light type, wavelength, power, and energy density; (3) sample size; (4) treatment protocol and location of laser application; (5) method of evaluation; (6) follow-up period; and (7) outcome of study.

To evaluate the quality of the included studies, the Jadad scale was used as previously described by Genev et al. ${ }^{38}$ The articles with 3 or more points were categorized as good quality studies and articles with less than 3 points were labeled as poor quality studies.

\section{Results and Discussion}

\section{Selection}

The PRISMA flow diagram of our search strategy in this study is illustrated in Fig. 1. Thirty-five articles were retrieved from Medline and 47 articles from other databases and 1 study from hand search. Two independent reviewers initially found 82 articles with screening, and 28 studies were excluded due to duplication and unrelated keywords according to our review design. After exclusion of nonrelevant articles, the full texts of 47 studies were evaluated. Within these studies, six of them were excluded since they 


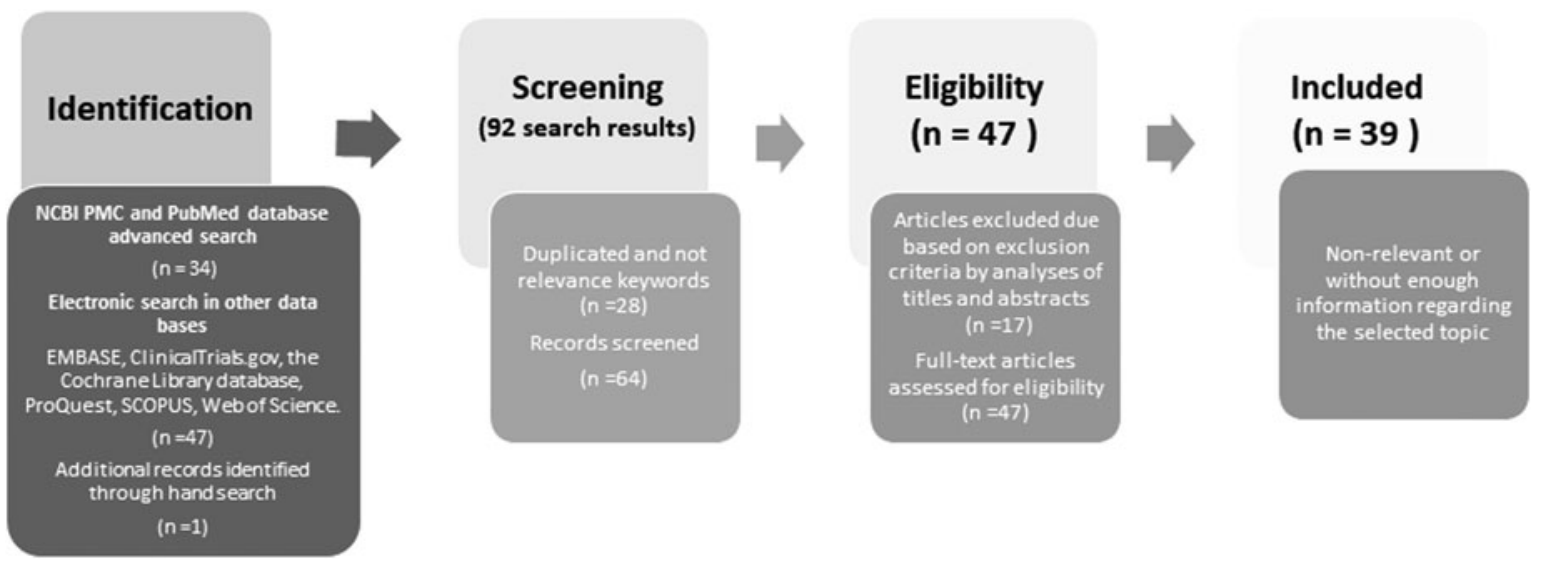

FIG. 1. Study design flowchart based on the PRISMA statement.

were review studies. ${ }^{2,39-43}$ One of the excluded studies was a case report ${ }^{44}$ and another study did not report light properties. ${ }^{45}$ Finally, 39 studies were selected and analyzed in this systematic review. All studies are summarized in Table 1.

\section{Indications of PBM for TMDs}

The findings of the included articles suggested that PBM was an effective way of decreasing pain as compared with placebo in TMD patients (29 studies out of 39). Moreover, PBM improved mandibular movements in eight studies ${ }^{46-54}$ and reduced patients' anxiety in two studies. ${ }^{46,48}$ Collecting information of these heterogeneous clinical trials showed beneficial impacts of PBM on reducing pain at the shortterm follow-up (10 days to 4 weeks) both in the visual analog scale $^{36}$ and in the anxiety questionnaire. ${ }^{46,47,55-57}$ Among these studies, GaAlAs diode at $780 \mathrm{~nm}$ wavelength and $50 \mathrm{~mW}$ power showed the most frequent nonsignificant effects. ${ }^{58,59}$ Functionality of TMJ was assessed in terms of maximum opening, electromyography, geometric mean diameter, colorimetric capsule method, and craniomandibular index, indicating that the total effect favored PBM in comparison with placebo. ${ }^{46-54}$ Moreover, four studies indicated pressure pain threshold improved by PBM, ${ }^{49,60-62}$ but De Carli et al. ${ }^{63}$ showed that $\mathrm{PBM}+$ piroxicam as well as piroxicam alone was as effective, but piroxicam was better for pain at 30 days. This fact leads us to the suggestion that the administration of PBM has to been seen as an adjuvant therapeutic approach for TMD treatment ${ }^{42,52}$ due to its analgesic, anti-inflammatory, and regenerative effects with high patients' satisfaction and minimum side effects. ${ }^{34,41,60,62-64}$ Most of the articles' Jadad scale numbers were 5 (highest score), and only four of them got 3 points due to the lack of blinding or blinding method, ${ }^{54,59,48}$ and randomization method, ${ }^{65}$ showing that all included articles had good quality.

Given the lack of robust evidence and also the heterogeneity of existing data regarding PBMT for TMDs, previous systematic reviews could not reach a conclusive protocol. $^{2,39,66,67}$ In the current review, we collect all existing clinical trials about the analgesic impact of PBMT that are inconsistent with previously reported reviews by $\mathrm{Xu}$ et al. ${ }^{66}$ and Chang et al. ${ }^{2}$ In addition, findings about the mandibular motion were in accordance with Chen et al. ${ }^{42}$ and $\mathrm{Xu}$ et al. ${ }^{66}$ Our systematic review, in comparison with the aforementioned reviews, covers a larger number of investigations and includes the most recent articles. Pain is the most common chief complaint of TMDs and the most frequent PBMT indication. This symptom can occur at any stage of TMDs and it has been shown that pain relief can ameliorate chewing and functionality of jaws and masticatory muscles. ${ }^{42,49,65,68}$ Thus, our first aim was to assess the impact of PBMT on pain alteration. Drawing a precise conclusion is difficult due to the diversity of the physical parameters of PBMT and various dosage application. For instance, there are four studies comparing low dosage application with high dosage. ${ }^{51,60,69,70}$ Only one of them ${ }^{60}$ demonstrated better results in high dosage application, whereas the others showed no difference. In this review, focus has been on dosage/fluence/energy density $\left(\mathrm{J} / \mathrm{cm}^{2}\right)$. Another important parameter in PBMT is the energy per point, which is less frequently reported but can in many cases be calculated. Other parameters such as time of irradiation, spot size, and beam configuration will influence the result and all these parameters need to be attended to in future research. For clinical studies, the World Association for Laser Therapy ${ }^{71}$ recommends the use of "energy dose", ( $\mathrm{J}$ ) and reporting in $\mathrm{J} / \mathrm{cm}^{2}$ should also be confined to in vitro and in vivo investigations. ${ }^{72}$

\section{PBM physical properties}

The physical properties of the irradiation such as wavelength, power, and energy density were different within the included studies in addition to a varying magnitude of energy applied on the target site. For instance, in the study by Carrasco et al., ${ }^{69}$ three different dosages were used: 25, 60, and $105 \mathrm{~J} / \mathrm{cm}^{2}$, but the findings were the same for all protocols. After analyzing the most frequent and successful protocols for PBM pain relief and movement enhancement, we suggest the best window of parameters for PBMT of TMD patients in Table 2. Fourteen studies reported how the actual energy output of the laser has been verified. $^{22,46,48-50,53,57,59,63,73-77}$ Authors are sometimes seemingly reporting properties according to manufacturer specification rather than performing independent measuring. We have summarized their calculated energy density, which was between 4 and $112.5 \mathrm{~J} / \mathrm{cm}^{2}$ as given in Table 1 . 


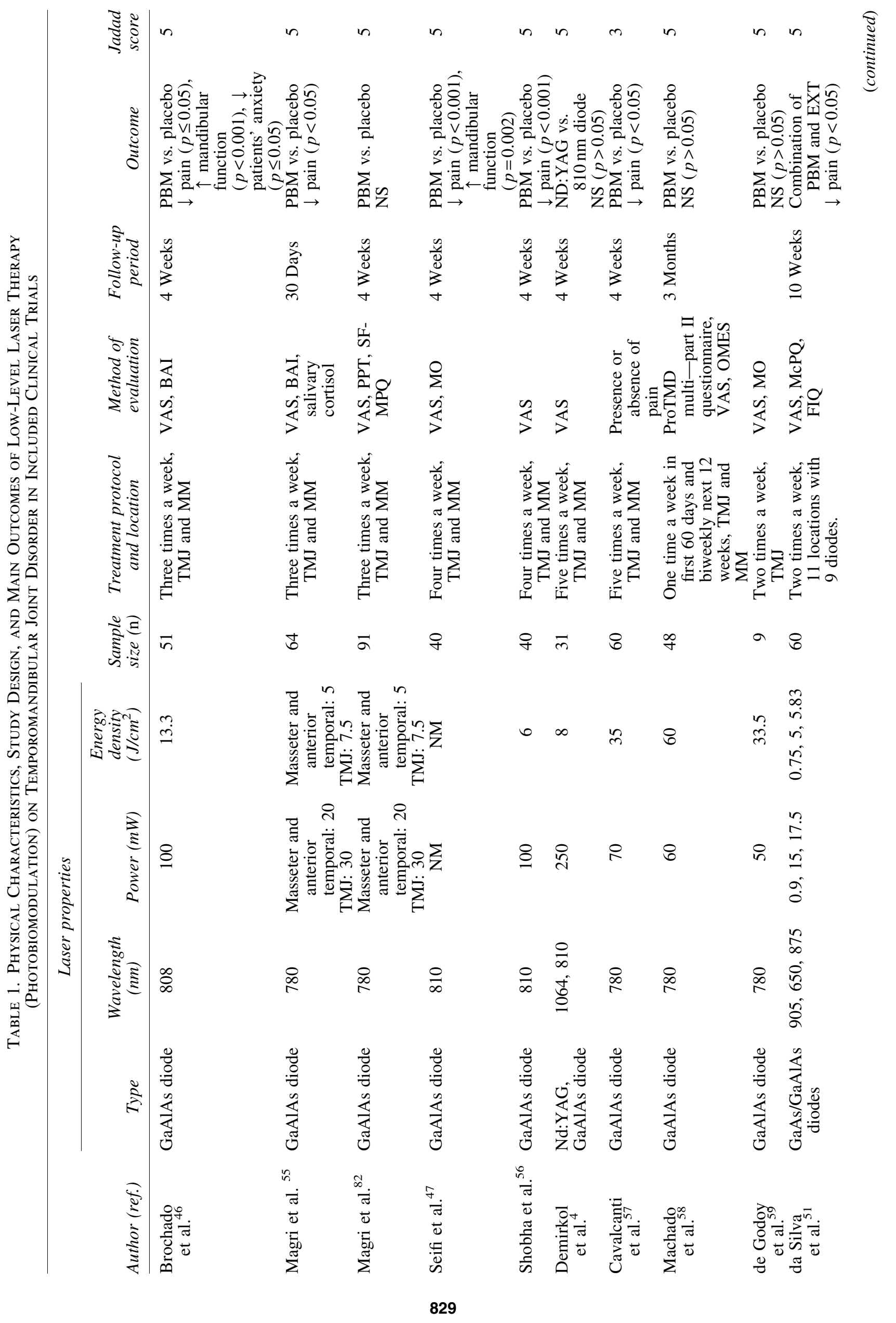




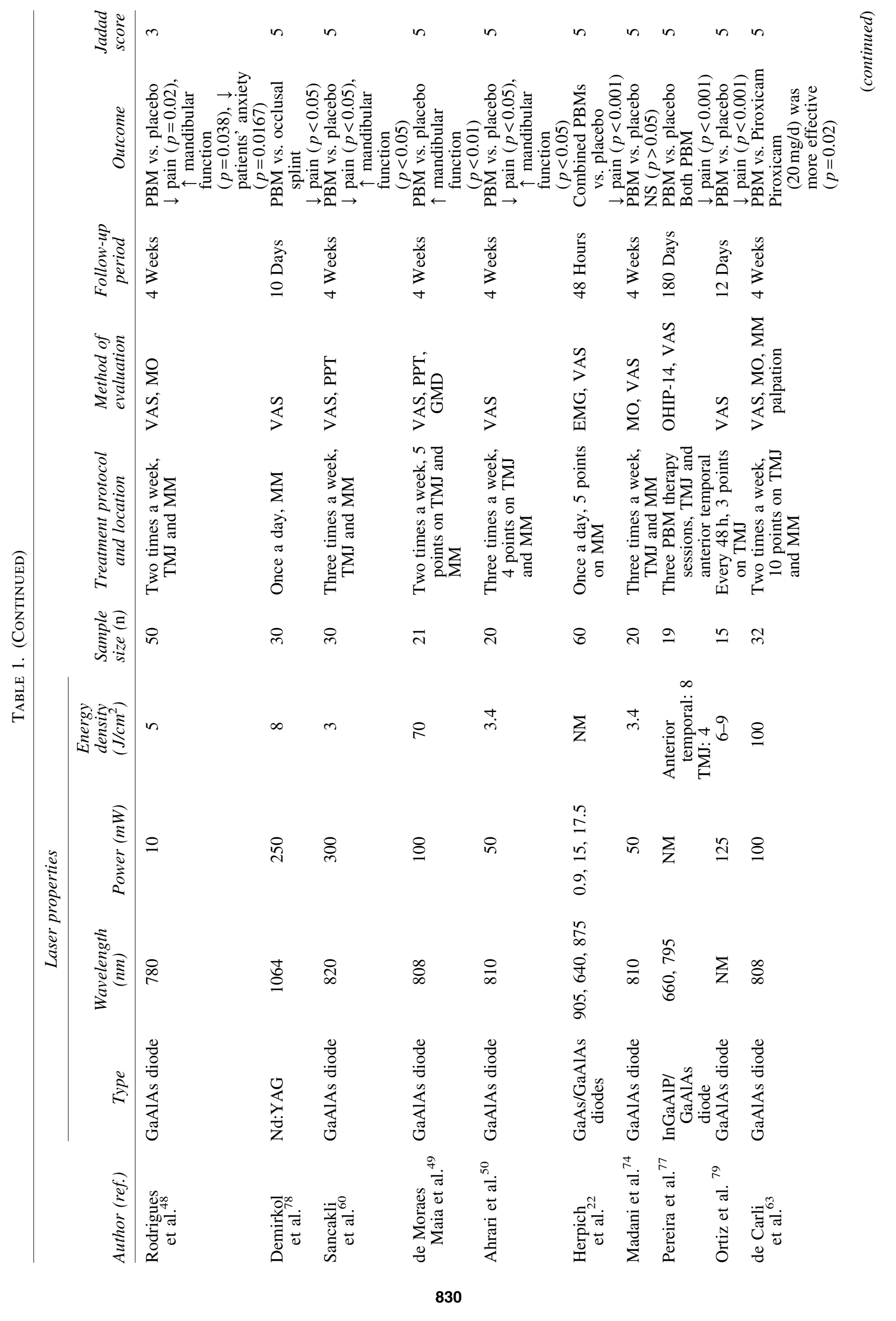




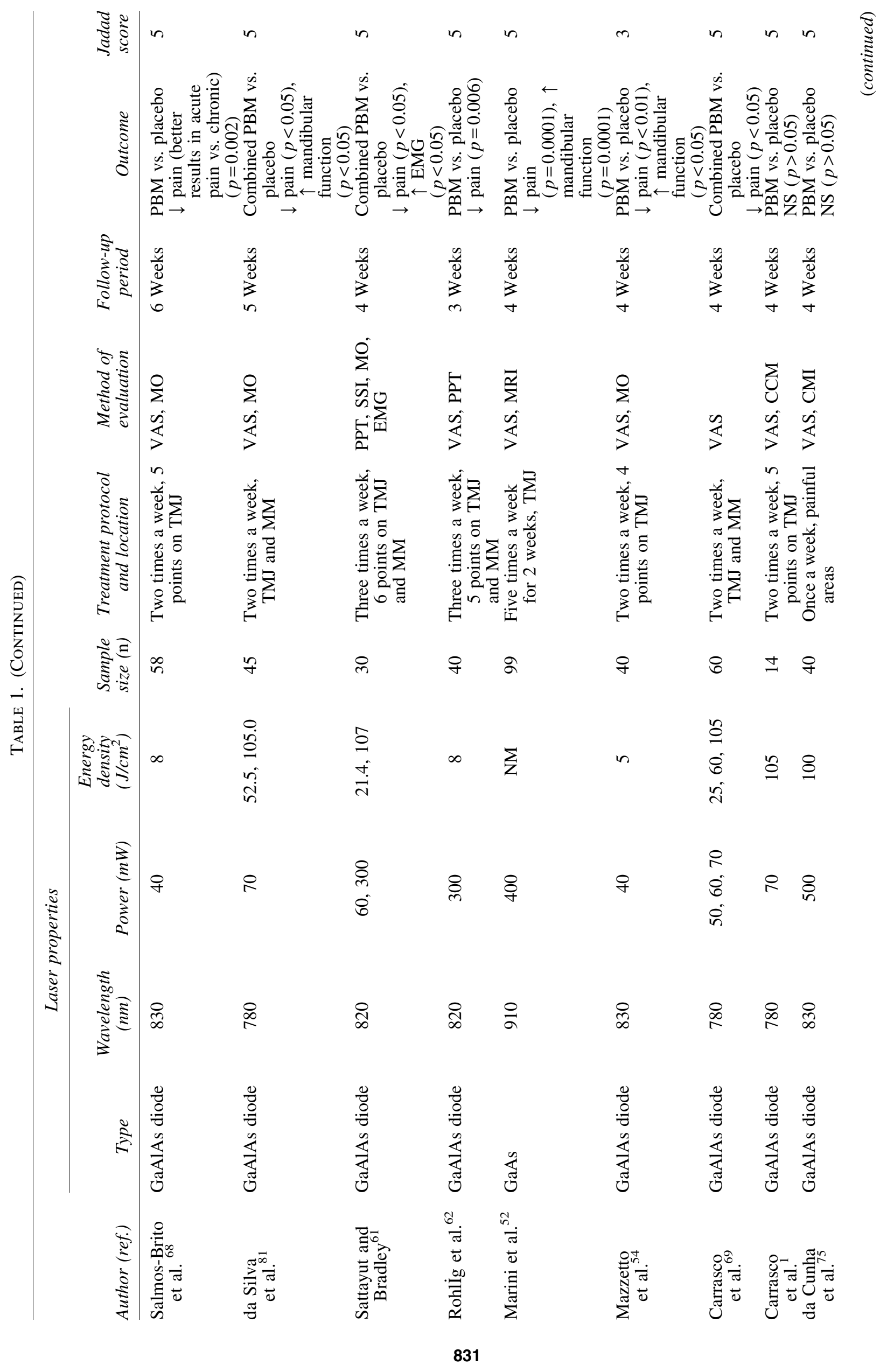




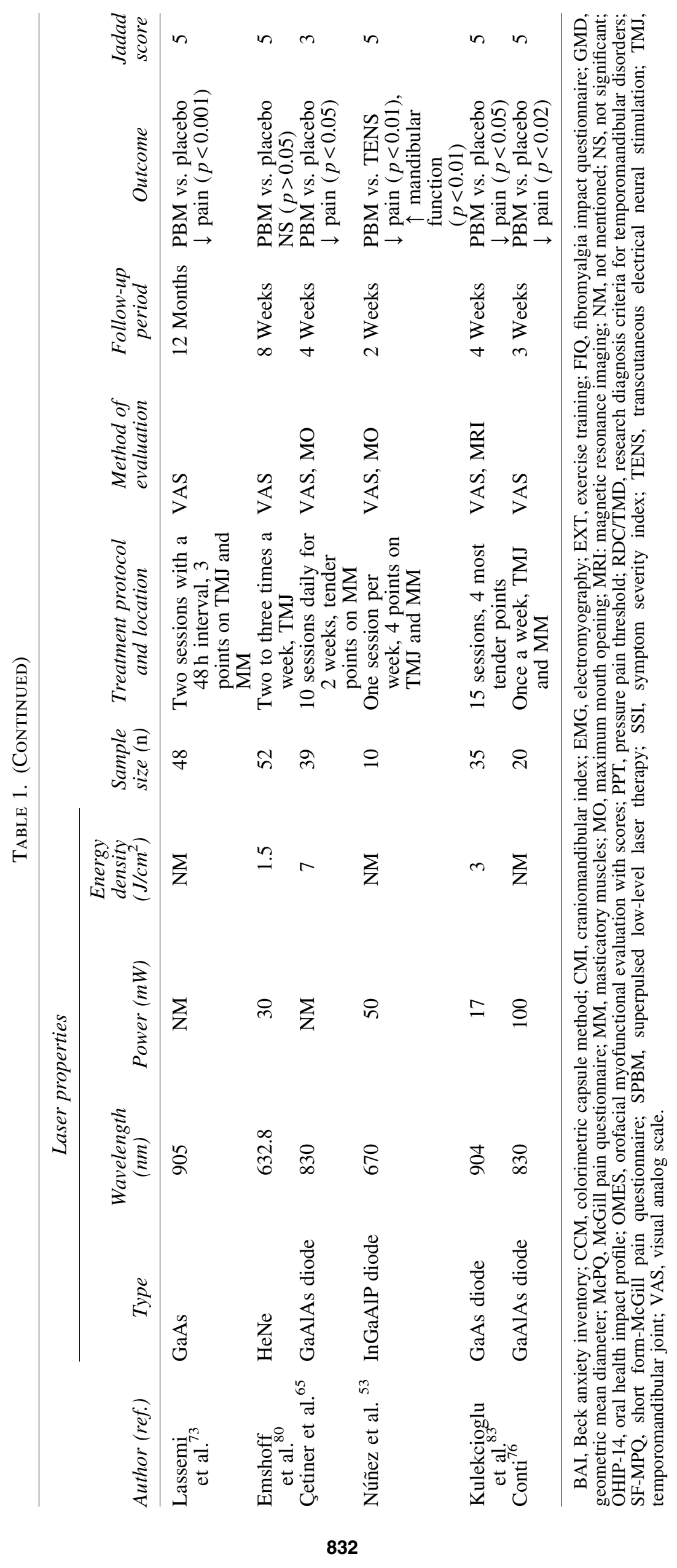


Table 2. Suggested Protocol for Application of Low-Level Laser Therapy in Temporomandibular Disorder Patients According to the Previous Reviewed Studies

\begin{tabular}{ll}
\hline Parameter & \\
\hline Laser type & Suggested amount \\
Wavelength & GaAlAs diode \\
Operating mode & $800-900 \mathrm{~nm}$ \\
Radiation power & Continuous \\
Energy density & $100-500 \mathrm{~mW}$ \\
No. of irradiation points & $<10 \mathrm{~J} / \mathrm{cm}^{2}$ \\
Session frequency and treatment duration & Includes all trigger points on masticatory muscles and TMJ \\
\hline
\end{tabular}

\section{PBM application site}

The location of PBM application varied between the included studies. PBM was applied to TMJ and masticatory muscles together in 25 studies, and to masticatory muscles only in 3 studies, ${ }^{22,65,78}$ and to TMJ only in 6 studies. $52,54,59,68,79,80$

Considering the nonsignificant results, which were more common among studies that irradiated only painful points or the TMJ, ${ }^{1,59,74}$ it seems that it is better to apply PBM on skin of both trigger points and TMJ. ${ }^{46-48,55-57}$ Moreover, according to the results of the included studies, PBMT is more effective when administered on the muscles compared with TMJ. But, Emshoff et al. ${ }^{80}$ in a well-designed study demonstrated similar effects of PBMT and placebo, which is in accordance with Herpich et al. ${ }^{22}$ One explanation, especially for Emshoff et al.'s ${ }^{80}$ findings, is their investigated patients. They included and treated patients with PBMT who had already been resistant to conventional therapeutic approaches for TMDs, such as diet change, occlusal appliances, or topical medicines. The use of HeNe laser may also influence the outcome, considering the limited penetration of this wavelength into muscles.

\section{PBM application protocols for TMDs}

The number of therapeutic sessions performed differed considerably, ranging from 10 sessions during 2 weeks in Çetiner et al. ${ }^{65}$ to once a week only, ${ }^{58,75,76,81}$ and the treatment duration varied from $10 \mathrm{sec}$ to $20 \mathrm{~min}$ for each session. It seems that more sessions did not promote PBM efficacy when comparing three different studies of same wavelength. ${ }^{58,59,82}$ In the latter studies, PBM was applied for one, two, or three sessions per week, but reported a nonsignificant difference. As aforementioned, the duration of PBMT is not the only important factor, but when PBM is administered only on the masticatory muscles, longer irradiation duration should be considered that leads to positive results. $^{80}$

\section{Considerations}

In this review, many included studies support the application of PBM for the treatment of TMDs (joint and/or muscles). ${ }^{1,46-51,58,59,77,80,82,83}$ Further, PBM is supported as an adjunct therapeutic modality for TMDs. Thus, more positive findings could probably be achieved by combining such therapy with standard TMD therapies. Further to that, no side effects were reported in the analyzed studies. For these reasons, researchers are encouraged to further investigate the capabilities of PBM to reach more consistent protocols in treating TMD cases.

\section{Future Direction and Conclusions}

Despite the observed limitation of PMBT, it seems that PBM can relieve pain in TMD patients and improve mandibular functionality. Given the high discrepancy within the included studies in this review, we highlight the need for further precise randomized clinical trials (RCTs) with larger sample sizes to assess its efficacy. More research must clarify the uncertain issues of PBM in TMDs such as dosage, energy, power density, duration, and locations. Further, future RCTs should include appropriate power output analysis, proper allocation concealment analysis, adequate randomization method, and double-blind design. Despite the variety of parameters related to the PBMT and the difficulty of their interpretation, an evidence-based consensus about the most efficient therapeutic protocols for TMD patients can be beneficial for both future research and clinical applications.

\section{Author Disclosure Statement}

No competing financial interests exist.

\section{Funding Information}

There was no funding provided for this article.

\section{References}

1. Carrasco TG, Mazzetto MO, Mazzetto RG, Mestriner W Jr. Low intensity laser therapy in temporomandibular disorder: a phase II double-blind study. Cranio 2008;26: 274-281.

2. Chang W-D, Lee C-L, Lin H-Y, Hsu Y-C, Wang C-J, Lai P-T. A meta-analysis of clinical effects of low-level laser therapy on temporomandibular joint pain. J Phys Ther Sci 2014;26:1297-1300.

3. Medlicott MS, Harris SR. A systematic review of the effectiveness of exercise, manual therapy, electrotherapy, relaxation training, and biofeedback in the management of temporomandibular disorder. Phys Ther 2006;86: 955-973.

4. Demirkol N, Usumez A, Demirkol M, Sari F, Akcaboy C. Efficacy of low-level laser therapy in subjective tinnitus patients with temporomandibular disorders. Photomed Laser Surg 2017;35:427-431.

5. Dahlström L, Carlsson GE. Temporomandibular disorders and oral health-related quality of life. A systematic review. Acta Odontol Scand 2010;68:80-85.

6. Oliveira LK, Almeida GdA, Lelis ER, Tavares M, Fernandes Neto AJ. Temporomandibular disorder and anxiety, 
quality of sleep, and quality of life in nursing professionals. Braz Oral Res 2015;29:1-7.

7. Schierz O, John MT, Reißmann DR, Mehrstedt M, Szentpétery A. Comparison of perceived oral health in patients with temporomandibular disorders and dental anxiety using oral health-related quality of life profiles. Qual Life Res 2008;17:857.

8. De Kanter R, Truin G, Burgersdijk R, et al. Prevalence in the Dutch adult population and a meta-analysis of signs and symptoms of temporomandibular disorder. J Dent Res 1993;72:1509-1518.

9. Jussila P, Kiviahde H, Näpänkangas R, et al. Prevalence of temporomandibular disorders in the Northern Finland Birth Cohort 1966. J Oral Facial Pain Headache 2017;31:159164.

10. Sampaio NdM, Oliveira MC, Ortega AdO, Santos LdB, Alves TDB. Temporomandibular disorders in elderly individuals: the influence of institutionalization and sociodemographic factors. Codas 2017;29:e20160114.

11. Chisnoiu AM, Picos AM, Popa S, et al. Factors involved in the etiology of temporomandibular disorders-a literature review. Clujul Med 2015;88:473.

12. Albertin A, Kerppers I, Amorim C, Costa R, Ferrari JC, Oliveira C. The effect of manual therapy on masseter muscle pain and spasm. Electroencephalogr Clin Neurophysiol 2010;50:107-112.

13. Sójka A, Huber J, Kaczmarek E, Hędzelek W. Evaluation of mandibular movement functions using instrumental ultrasound system. J Prosthodont 2017;26:123-128.

14. Rodrigues D, Siriani AO, Bérzin F. Effect of conventional TENS on pain and electromyographic activity of masticatory muscles in TMD patients. Braz Oral Res004;18:290 295.

15. Gomes NC, Berni-Schwarzenbeck K, Packer AC, Rdrigues-Bigaton D. Effect of cathodal high-voltage electrical stimulation on pain in women with TMD. Braz J Phys Ther 2012;16:10-15.

16. Al-Ani Z, Gray R. TMD current concepts: 1. An update. Dental update 2007;34:278-288.

17. Bertolucci LE, Grey T. Clinical analysis of mid-laser versus placebo treatment of arthralgic TMJ degenerative joints. Cranio 1995;13:26-29.

18. Dolwick MF. The role of temporomandibular joint surgery in the treatment of patients with internal derangement. Oral Surg Oral Med Oral Pathol Oral Radiol Endod 1997;83: 150-155.

19. Mussttaf RA, Jenkins DF, Jha AN. Assessing the impact of low level laser therapy (LLLT) on biological systems: a review. Int J Radiat Biol 2019;95:120-143.

20. Heiskanen V, Hamblin MR. Photobiomodulation: lasers vs. light emitting diodes? Photochem Photobiol Sci 2018;17: 1003-1017.

21. Panhoca VH, Lizarelli RdFZ, Nunez SC, et al. Comparative clinical study of light analgesic effect on temporomandibular disorder (TMD) using red and infrared led therapy. Lasers Med Sci 2015;30:815-822.

22. Herpich CM, Leal-Junior ECP, Gomes CAF, et al. Immediate and short-term effects of phototherapy on pain, muscle activity, and joint mobility in women with temporomandibular disorder: a randomized, double-blind, placebo-controlled, clinical trial. Disabil Rehabil 2018;40: 2318-2324.

23. Farivar S, Malekshahabi T, Shiari R. Biological effects of low level laser therapy. Lasers Med Sci 2014;5:58.
24. Javed F, Kellesarian S, Romanos G. Role of diode lasers in oro-facial pain management. J Biol Regul Homeost Agents 2017;31:153-155.

25. Chow RT, Armati PJ. Photobiomodulation: implications for anesthesia and pain relief. Photomed Laser Surg 2016;34: 599-609.

26. Honmura A, Ishii A, Yanase M, Obata J, Haruki E. Analgesic effect of Ga-Al-As diode laser irradiation on hyperalgesia in carrageenin-induced inflammation. Lasers Surg Med 1993;13:463-469.

27. Sakurai Y, Yamaguchi M, Abiko Y. Inhibitory effect of low-level laser irradiation on LPS-stimulated prostaglandin E2 production and cyclooxygenase-2 in human gingival fibroblasts. Eur J Oral Sci 2000;108:29-34.

28. Moseley AM, Herbert RD, Sherrington C, Maher CG. Evidence for physiotherapy practice: a survey of the Physiotherapy Evidence Database (PEDro). Aust J Physiother 2002;48:43-49.

29. Bayat M, Virdi A, Rezaei F, Chien S. Comparison of the in vitro effects of low-level laser therapy and low-intensity pulsed ultrasound therapy on bony cells and stem cells. Prog Biophys Mol Biol 2018;133:36-48.

30. Matsumoto MA, Ferino RV, Monteleone GF, Ribeiro DA. Low-level laser therapy modulates cyclo-oxygenase-2 expression during bone repair in rats. Lasers Med Sci 2009; 24:195-201.

31. Tim CR, Pinto KNZ, Rossi BRO, et al. Low-level laser therapy enhances the expression of osteogenic factors during bone repair in rats. Lasers Med Sci 2014;29:147-156.

32. Tim CR, Bossini PS, Kido HW, et al. Effects of low level laser therapy on inflammatory and angiogenic gene expression during the process of bone healing: a microarray analysis. J Photochem Photobiol B 2016;154:8-15.

33. Petrucci A, Sgolastra F, Gatto R, Mattei A, Monaco AJ. Effectiveness of low-level laser therapy in temporomandibular disorders: a systematic review and meta-analysis. J Orofac Pain 2011;25:298.

34. Vladimirov YA, Osipov A, Klebanov GJB. Photobiological principles of therapeutic applications of laser radiation. Biochemistry (Moscow) 2004;69:81-90.

35. Moher D, Shamseer L, Clarke M, et al. Preferred reporting items for systematic review and meta-analysis protocols (PRISMA-P) 2015 statement. Syst Rev 2015;4:1.

36. Pandeshwar P, Roa MD, Das R, Shastry SP, Kaul R, Srinivasreddy MB. Photobiomodulation in oral medicine: a review. J Investig Clin Dent 2016;7:114-126.

37. Miura M, Gronthos S, Zhao M, et al. SHED: stem cells from human exfoliated deciduous teeth. Proc Natl Acad Sci U S A 2003;100:5807-5812.

38. Genev IK, Tobin MK, Zaidi SP, Khan SR, Amirouche FM, Mehta AI. Spinal compression fracture management: a review of current treatment strategies and possible future avenues. Global Spine J 2017;7:71-82.

39. Zokaee H, Zahmati AHA, Mojrian N, Boostani A, Vaghari M. Efficacy of low-level laser therapy on orofacial pain: a literature review. Adv Hum Biol 2018;8:70.

40. Shukla D, Muthusekhar M. Efficacy of low-level laser therapy in temporomandibular disorders: a systematic review. Natl J Maxillofac Surg 2016;7:62.

41. Herranz-Aparicio J, Vázquez-Delgado E, ArnabatDomínguez J, España-Tost A, Gay-Escoda C. The use of low level laser therapy in the treatment of temporomandibular joint disorders. Review of the literature. Med Oral Patol Oral Cir Bucal 2013;18:e603. 
42. Chen J, Huang Z, Ge M, Gao M. Efficacy of low-level laser therapy in the treatment of TMDs: a meta-analysis of 14 randomised controlled trials. J Oral Rehab 2015;42:291299.

43. Maia MLdM, Bonjardim LR, Quintans JdSS, Ribeiro MAG, Maia LGM, Conti PCR. Effect of low-level laser therapy on pain levels in patients with temporomandibular disorders: a systematic review. J Appl Oral Sci 2012;20: 594-602.

44. Ayyildiz S, Emir F, Sahin C. Evaluation of low-level laser therapy in TMD patients. Case Report Dent 2015;2015: 424213.

45. Melchior MdO, Machado BCZ, Magri LV, Mazzetto MO. Effect of speech-language therapy after low-level laser therapy in patients with TMD: a descriptive study. Codas 2016;28:818-822.

46. Brochado FT, Jesus LH, Carrard VC, Freddo AL, Chaves $\mathrm{KD}$, Martins MD. Comparative effectiveness of photobiomodulation and manual therapy alone or combined in TMD patients: a randomized clinical trial. Braz Oral Res 2018;32:e50.

47. Seifi M, Ebadifar A, Kabiri S, Badiee MR, Abdolazimi Z, Amdjadi P. Comparative effectiveness of low level laser therapy and transcutaneous electric nerve stimulation on temporomandibular joint disorders. J Lasers Med Sci 2017; 8:S27.

48. Rodrigues JH, Marques MM, Biasotto-Gonzalez DA, et al. Evaluation of pain, jaw movements, and psychosocial factors in elderly individuals with temporomandibular disorder under laser phototherapy. Lasers Med Sci 2015;30: 953-959.

49. de Moraes Maia ML, Ribeiro MAG, Maia LGM, et al. Evaluation of low-level laser therapy effectiveness on the pain and masticatory performance of patients with myofascial pain. Lasers Med Sci 2014;29:29-35.

50. Ahrari F, Madani AS, Ghafouri ZS, Tunér J. The efficacy of low-level laser therapy for the treatment of myogenous temporomandibular joint disorder. Lasers Med Sci 2014; 29:551-557.

51. da Silva MM, Albertini R, de Carvalho PD, et al. Randomized, blinded, controlled trial on effectiveness of photobiomodulation therapy and exercise training in the fibromyalgia treatment. Lasers Med Sci 2018;33:343-351.

52. Marini I, Gatto MR, Bonetti GA. Effects of superpulsed low-level laser therapy on temporomandibular joint pain. Clin J Pain 2010;26:611-616.

53. Núñez SC, Garcez AS, Suzuki SS, Ribeiro MS. Management of mouth opening in patients with temporomandibular disorders through low-level laser therapy and transcutaneous electrical neural stimulation. Photomed Laser Surg 2006;24:45-49.

54. Mazzetto MO, Hotta TH, Pizzo RC. Measurements of jaw movements and TMJ pain intensity in patients treated with GaAlAs laser. Braz Dent J 2010;21:356-360.

55. Magri LV, Carvalho VA, Rodrigues FCC, Bataglion C, Leite-Panissi CRA. Non-specific effects and clusters of women with painful TMD responders and non-responders to LLLT: double-blind randomized clinical trial. Lasers Med Sci 2018;33:385-392.

56. Shobha R, Narayanan VS, Pai BJ, Jaishankar H, Jijin M. Low-level laser therapy: a novel therapeutic approach to temporomandibular disorder-a randomized, doubleblinded, placebo-controlled trial. Indian J Dent Res 2017; 28:380.
57. Cavalcanti MFXB, Silva UH, Leal-Junior ECP, et al. Comparative study of the physiotherapeutic and drug protocol and low-level laser irradiation in the treatment of pain associated with temporomandibular dysfunction. Photomed Laser Surg 2016;34:652-656.

58. Machado BCZ, Mazzetto MO, Da Silva MA, de Felício CM. Effects of oral motor exercises and laser therapy on chronic temporomandibular disorders: a randomized study with follow-up. Lasers Med Sci 2016;31:945-954.

59. de Godoy CHL, Motta LJ, Fernandes KPS, MesquitaFerrari RA, Deana AM, Bussadori SK. Effect of low-level laser therapy on adolescents with temporomandibular disorder: a blind randomized controlled pilot study. J Oral Maxillofac Surg 2015;73:622-629.

60. Sancakli E, Gökçen-Röhlıg B, Balık A, Öngül D, Kıpırdı S, Keskın H. Early results of low-level laser application for masticatory muscle pain: a double-blind randomized clinical study. BMC Oral Health 2015;15:131.

61. Sattayut S, Bradley P. A study of the influence of low intensity laser therapy on painful temporomandibular disorder patients. Laser Therapy 2012;21:183-192.

62. Rohlİg BG, Kipirdi S, Capan N, Keskİn H. Masticatory muscle pain and low-level laser therapy: a double-blind and placebo-controlled study. Turk J Phys Med Rehab 2011;57: 31-37.

63. De Carli M, Guerra M, Nunes T et al. Piroxicam and laser phototherapy in the treatment of TMJ arthralgia: a doubleblind randomised controlled trial. J Oral Rehab 2013;40: 171-178.

64. Shirani AM, Gutknecht N, Taghizadeh M, Mir M. Lowlevel laser therapy and myofacial pain dysfunction syndrome: a randomized controlled clinical trial. Lasers Med Sci 2009;24:715-720.

65. Çetiner S, Kahraman SA, Yücetas Ş. Evaluation of lowlevel laser therapy in the treatment of temporomandibular disorders. Photomed Laser Surg 2006;24:637-641.

66. Xu G-Z, Jia J, Jin L, Li J-H, Wang Z-Y, Cao D-Y. Lowlevel laser therapy for temporomandibular disorders: a systematic review with meta-analysis. Pain Res Manag 2018;2018:4230583.

67. Melis M, Di Giosia M, Zawawi KH. Low level laser therapy for the treatment of temporomandibular disorders: a systematic review of the literature. Cranio 2012;30:304-312.

68. Salmos-Brito JAL, de Menezes RF, Teixeira CEC, et al. Evaluation of low-level laser therapy in patients with acute and chronic temporomandibular disorders. Lasers Med Sci 2013;28:57-64.

69. Carrasco TG, Guerisoli LDC, Guerisoli DMZ, Mazzetto MO. Evaluation of low intensity laser therapy in myofascial pain syndrome. Cranio 2009;27:243-247.

70. Venezian GC, Antônio Moreira Rodrigues da Silva M, Mazzetto RG, Oliveira Mazzetto M. Low level laser effects on pain to palpation and electromyographic activity in TMD patients: a double-blind, randomized, placebocontrolled study. Cranio 2010;28:84-91.

71. World Association of Laser Therapy (WALT). Standards for the design and conduct of systematic reviews with lowlevel laser therapy for musculoskeletal pain and disorders. Photomed Laser Surg 2006;24:759-760.

72. World Association of Laser Therapy (WALT). Consensus agreement on the design and conduct of clinical studies with low-level laser therapy and light therapy for musculoskeletal pain and disorders. Photomed Laser Surg 2006; 24:761-762. 
73. Lassemi E, Jafari SM, Motamedi MHK, Navi F, Lasemi R. Low-level laser therapy in the management of temporamandibular joint disorder. J Oral Laser Appl 2008;8:83-86.

74. Madani AS, Ahrari F, Nasiri F, Abtahi M, Tunér J. Lowlevel laser therapy for management of TMJ osteoarthritis. Cranio 2014;32:38-44.

75. da Cunha LA, Firoozmand LM, da Silva AP, Esteves SA, de Oliveira W. Efficacy of low-level laser therapy in the treatment of temporomandibular disorder. Int Dent J 2008; 58:213-217.

76. Conti PCR. Low level laser therapy in the treatment of temporomandibular disorders (TMD): a double-blind pilot study. Cranio 1997;15:144-149.

77. Pereira TS, Flecha OD, Guimaraes RC et al. Efficacy of red and infrared lasers in treatment of temporomandibular disorders - a double-blind, randomized, parallel clinical trial. Cranio 2014;32:51-56.

78. Demirkol N, Sari F, Bulbul M, Demirkol M, Simsek I, Usumez A. Effectiveness of occlusal splints and low-level laser therapy on myofascial pain. Lasers Med Sci 2015;30: 1007-1012.

79. Ortiz MB, De Santillana IAE, Arellano JC. Effectiveness of low-level laser as a treatment for pain management in arthrogenic temporomandibular disorders. IDJSR 2013;1:67-73.

80. Emshoff R, Bösch R, Pümpel E, Schöning H, Strobl H. Low-level laser therapy for treatment of temporomandibular joint pain: a double-blind and placebo-controlled trial. Oral Surg Oral Med Oral Pathol Oral Radiol Endod 2008; 105:452-456.
81. da Silva MA, Botelho AL, Turim CV, da Silva AM. Low level laser therapy as an adjunctive technique in the management of temporomandibular disorders. Cranio 2012;30: 264-271.

82. Magri LV, Carvalho VA, Rodrigues FCC, Bataglion C, Leite-Panissi CRA. Effectiveness of low-level laser therapy on pain intensity, pressure pain threshold, and SF-MPQ indexes of women with myofascial pain. Lasers Med Sci 2017;32:419-428.

83. Kulekcioglu S, Sivrioglu K, Ozcan O, Parlak M. Effectiveness of low-level laser therapy in temporomandibular disorder. Scand J Rheumatol 2003;32:114-118.

Address correspondence to: Reza Fekrazad, DDS, PhD, FLD, FICD Radiation Sciences Research Center Lasers Research Center in Medical Sciences AJA University of Medical Sciences Flat No. 12, Mooj Building First Behestan, Pasdaran St Tehran 1946883893

Iran

E-mail: rezafekrazad@gmail.com

Received: June 2, 2019

Accepted after revision: August 2, 2019 Published online: November 27, 2019. 